\title{
Observable effects from spacetime tunneling
}

\author{
Pedro F. González-Díaz \\ Centro de Física “Miguel Catalán,”, Instituto de Matemáticas y Física Fundamental, Consejo Superior de Investigaciones Científicas, \\ Serrano 121, 28006 Madrid, Spain
}

(Received 19 March 1997)

\begin{abstract}
Assuming that spacetime tunnels_-wormholes and ringholes—naturally exist in the Universe, we investigate the conditions making them embeddible in Friedmann space, and the possible observable effects of these tunnels, including lensing and frequency shifting of emitting sources, discontinuous change of background temperature, broadening and intensity enhancement of spectral lines, as well as a dramatic increase of the luminosity of any object at the tunnel's throat. [S0556-2821(97)02322-9]
\end{abstract}

PACS number(s): 04.20.Gz, 98.80.Es, 98.80.Hw

\section{INTRODUCTION}

Solutions to Einstein equations corresponding to spacetimes with closed timelike curves (CTC's) have stirred the relativistics, following developments by Lanczos [1], van Stockum [2], Gödel [3], Misner [4], and, more recently, Morris and Thorne [5], Gott [6], and Jensen and Soleng [7]. The reason for the excitement and subsequent general disbelief resided much in that, being consistent solutions to Einstein equations for very special kinds of matter, the proposed spacetimes allow for the possibility of time travel and, hence for potential violations of causality [8]. Here we look at the idea that, rather than being interpreted as constructs to be eventually built up from future highly developed technology, spacetime tunnels generating CTC's may spontaneously exist in some regions of our universe, and give rise to observable effects which could be detected even with present technology.

Thus, we consider CTC's generated along spacetime tunnels whose mouths embed in distant regions with the Friedmann geometry of the overall universe. The necessary condition for such tunnels to occur in a given region is that, in that region there is a certain proportion of matter with negative energy $[8,9]$. Two tunnel topologies have been considered so far. That of a two-sphere which gives rise to wormholes $[5,10]$, and that of a two-torus which is associated with the so-called ringholes [11]. Both tunneling types are traversable and convertible into time machines generating CTC's by simply letting one of the hole's mouths to move toward the other $[5,10,11]$, but whereas the energy density is everywhere negative near the throat of a wormhole, it still becomes positive for values of the angle $\varphi_{2}$ (defining the position on the surface circles determined by the torus sections) such that $2 \pi-\varphi_{h}>\varphi_{2}>\varphi_{h}$, with $\varphi_{h}=\arccos (b / a)$, where $a$ and $b$ are the radius of the circumference generated by the circular axis of the torus and that of a torus section, respectively, at the ringhole's throat [11].

The purpose of the present work is to investigate under what conditions can a tunnel be embedded in a cosmological spacetime, and explore the effects that the inner properties of spacetime tunnels may have on the observable characteristics of astronomical objects placed beyond or passing through these tunnels, relative to an observer whose line of sight to the object traverses or does not traverse the given tunnel.
Most of the emphasis will be placed on ringholes, but the results will always be compared with those expected from wormholes.

\section{EMBEDDING A TUNNEL IN FRIEDMANN SPACE}

Assuming that the amount of negative mass equals that of positive mass, the gravitational field that represents tunneling through a traversable ringhole can be described by a spacetime metric [11]:

$$
d s^{2}=-d t^{2}+\left(\frac{n}{r}\right)^{2} d l^{2}+m^{2} d \varphi_{1}^{2}+b^{2} d \varphi_{2}^{2}
$$

where $-\infty<t<\infty, l$ is the proper radial distance of each transversal section of the ringhole's torus, $-\infty<l<\infty, \varphi_{1}$ and $\varphi_{2}$ are as given for the torus metric [11], and

$$
\begin{gathered}
m=a-b \cos \varphi_{2}, \quad n=b-a \cos \varphi_{2}, \\
r=\sqrt{a^{2}+b^{2}-2 a b \cos \varphi_{2}} .
\end{gathered}
$$

There are two $\varphi_{2}$-angular horizons. They occur at $\varphi_{2}=\varphi_{h}=\arccos (b / a)$ and $2 \pi-\varphi_{h}$. For $-\varphi_{h}<\varphi_{2}<\varphi_{h}$, the ringhole would behave like a converging lens near the throat, and for $2 \pi-\varphi_{h}>\varphi_{2}>\varphi_{h}$, the ringhole would behave like the wormhole; i.e., it acts as a diverging lens near the throat. This property may be crucial to identify the existence of these holes in the Universe and distinguish between them.

Metric (2.1) can easily be converted into that of a spherical wormhole by applying the coordinate change [11] $a \rightarrow 0$, $\varphi_{2} \rightarrow \theta+\pi / 2, \varphi_{1} \rightarrow \phi$, and allowing a given purely negative mass $M$ to induce a general factor $e^{2 \Phi(r, M)}$ in the $g_{t t}$ components of the metric tensor.

For a section of constant $\varphi_{1}$ and $\varphi_{2}$, the ringhole metric (2.1) can be written as

$$
d s^{2}=b^{2}\left(-d \eta^{2}+d \chi^{2}\right)
$$

where

$$
d \eta=\frac{d t}{b}, \quad d \chi=\frac{n d l}{r b} .
$$


In the approximation in which $b \gg b_{0}$ (where $b_{0}$ is the value of $b$ at the throat), $l \sim b$ [11], one can integrate the second of expressions (2.4) to

$$
\chi=\chi_{0}+\ln \left[\left(\frac{r}{a}+\frac{b}{a}-\cos \varphi_{2}\right)\left(\frac{a}{b}+\frac{r}{b}-\cos \varphi_{2}\right)^{\cos \varphi_{2}}\right] .
$$

When a null geodesic is considered, $d s=0$ and we obtain

$$
\begin{gathered}
b^{\prime}=\frac{d b}{d \eta}=b \frac{d b}{d t}=\frac{r l}{n}, \\
d \eta= \pm d \chi,
\end{gathered}
$$

or integrating

$$
\chi= \pm \eta+\chi_{0}
$$

and hence, following the same line of reasoning as in, e.g., Ref. [12], we obtain that the frequency of the light rays is either redshifted while traveling from mouth to throat by an amount

$$
z=\frac{\omega-\omega_{0}}{\omega_{0}}=-\chi \frac{b^{\prime}}{b}=-\chi \frac{r l}{n b},
$$

or blue-shifted by exactly the same amount if the light rays travel from throat to mouth. Therefore, along complete passage through a static ringhole, the frequency of a light ray preserves its initial value. However, the embedding of the ringhole mouths in two respectively distant regions of a Friedmann spacetime with scale factor $R\left(\eta_{F}\right)$, conformal time $\eta_{F}=\int\left(d t_{F} / R\right)$ and distance from the origin $R \chi_{F}$, ensures that such two distant regions are redshifted relative to one another by the known cosmological amount predicted from $\omega=\omega_{0} R\left(\eta_{F}-\chi_{F}\right) / R\left(\eta_{F}\right)$ [12], both for static ringholes and wormholes. It follows that these tunnels cannot embed in the background Friedmann spacetime, unless for the case that the tunnels are allowed to be no longer static, with one of the hole's mouths moving with respect to the other mouth with a nonzero velocity, $V$, such that the Doppler shift resulting from this motion would equal the usual cosmological redshift:

$$
z=-\frac{H D}{c}=-\frac{V}{c}
$$

with $H$ Hubble's constant and $D$ the distance, both for the wormhole and ringhole. In the latter case, the initial distance between mouths can be calculated to be

$$
L_{r}=2 \int \frac{n b}{r l} d b=2 a,
$$

where we have discarded the integration constant, so that Eq. (2.8) can finally be rewritten as

$$
z=-\frac{H D}{c}-\frac{2 H_{r} a}{c}
$$

with $H_{r}=b^{\prime} / b^{2}$.
The metric of a ringhole whose mouths move with velocity $V$ relative to one another has the form [11]

$$
d s^{2}=-\left[1+g l F(l) \sin \varphi_{2}\right]^{2} d t^{2}+d l^{2}+m^{2} d \varphi_{1}^{2}+b^{2} d \varphi_{2}^{2},
$$

where $g=\gamma^{2}(d V / d t), \quad \gamma=1 / \sqrt{1-V^{2}}, l=\sqrt{b^{2}-b_{0}^{2}}$, with $b_{0}$ the value of $b$ at the throat, and $F(l)$ is a form factor that, if we assume mouth $A$ to be moving, vanishes in the half of the ringhole with the other mouth, and rises monotonously from 0 to 1 as one moves from the throat to mouth $A$. For this ringhole to be embedded in Friedmann spacetime, the velocity $V$ must be restricted to be

$$
V=H D=\frac{\dot{R}}{R} D,
$$

with the overhead dot meaning derivative respect to the Friedmann time $t_{F}$. In this case, the embedding of sections in the ringhole space with constant angles $\varphi_{1}$ and $\varphi_{2}$ in sections of Friedmann space with constant angles $\theta$ and $\phi$, implies

$$
d t_{F}=\left[1+g l F(l) \sin \varphi_{2}^{(0)}\right] d t
$$

with $t_{F}$ and $t$ the times for, respectively, Friedmann and ringhole spacetimes.

Let us evaluate $g$ at given constant values of $\varphi_{2}^{(0)}$ and $D$. We have

$$
g=\gamma^{2} \frac{d V}{d t}=\gamma^{2} D\left(\frac{d H}{d t_{F}}\right)\left[1+g l F(l) \sin \varphi_{2}^{(0)}\right],
$$

where Eq. (2.13) has been used. From this we obtain

$$
g=\frac{\gamma^{2} D H^{2}(q-1)}{1-\gamma^{2} D H^{2}(q-1) l F(l) \sin \varphi_{2}^{(0)}},
$$

where $q=-\ddot{R} R / \dot{R}^{2}$ is the deceleration parameter at the time when the ringhole is formed. Hence, we obtain as the metric of a ringhole embeddible in Friedmann space:

$$
\begin{aligned}
d s^{2}= & -\left[1-\frac{D H^{2}(q-1) l F(l) \sin \varphi_{2}^{(0)}}{\left(1-H^{2} D^{2}\right)^{2}}\right]^{-2} d t^{2}+d l^{2}+m^{2} d \varphi_{1}^{2} \\
& +b^{2} d \varphi_{2}^{2}
\end{aligned}
$$

The corresponding embeddible metric for wormholes can again be obtained from this by using the transformation [11] $a \rightarrow 0, \varphi_{2} \rightarrow \theta+\pi / 2, \varphi_{1} \rightarrow \phi$, and introducing a factor $e^{2 \Phi}$ in the $g_{t t}$ component of the metric tensor. On the other hand, a ringhole embeddible in Friedmann space will develop closed timelike curves and hence convert into time machine at sufficiently late times, provided that the time shift induced by relative motion between mouths exceeds the distance between mouths [9], i.e.,

$$
t_{F}>\left|\frac{1-H^{2} D^{2}}{(q-1) H l F(l) H_{r} \sin \varphi_{2}^{(0)}}\right|,
$$

where Eqs. (2.10), (2.13), and (2.14) have been used. Thus, for a given time $t_{F}$, and provided that there exists a certain 


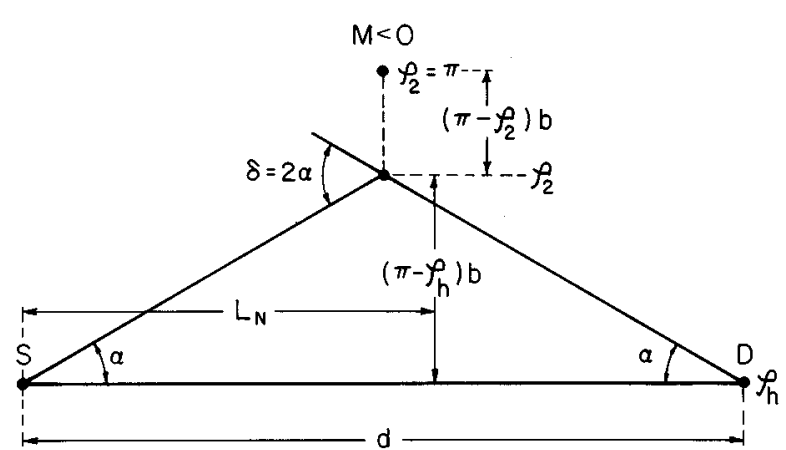

FIG. 1. Geometry for gravitational lensing by the negative mass placed at the throat of a ringhole. Off-axis light rays from stellar source $S$ are deflected to detector $D$ by the gravitational repulsion of negative mass $M$. For embeddible ringholes the two angles denoted $\alpha$ would become slightly different.

proportion of matter with negative energy, a ringhole time machine can be spontaneously created for the set of parameters $l, F(l), H_{r}$, and $\varphi_{2}^{(0)}$ which satisfy Eq. (2.16), so that any astronomical object going through the ringhole should traverse CTC's in the inner nonchronal region of the ringhole to travel backward or forward in time, depending on whether it enters the ringhole by its moving or stationary mouth [11].

\section{OBSERVABLE EFFECTS}

\section{A. Lensing}

Let us assume that at one of the ringhole's mouths there is a source of radiation which, since the hole is traversable, can be detected by an observer placed at the other mouth. Some of the rays emitted by the source will cross the ringhole's throat on one of the angular horizons, $\varphi_{h}$ say, where they will not undergo any deflection [11]. Other rays will, however, cross the throat along values of the angle $\varphi_{2}$ such that $2 \pi-\varphi_{h}>\varphi_{2}>\varphi_{h}$ and, therefore, will be deflected towards the angular horizon by gravitational repulsion of the negative energy placed on this horizon side. One should then expect that the observer will detect a double image of the source, such as it is described in Fig. 1. Assuming that the total amount of negative mass is $M<0$, we then have

$$
\delta=2 \alpha=\frac{4 G|M|}{b\left(\pi-\varphi_{2}\right) c^{2}} .
$$

Besides, for small $\alpha$ it can be obtained

$$
\alpha=\frac{\varphi_{2}-\varphi_{h}}{L_{N}} b=\frac{\varphi_{2}-\varphi_{h}}{d} 2 b .
$$

From Eqs. (3.1) and (3.2),

$$
\epsilon^{2}-\left(\epsilon_{h}+\epsilon_{\pi}\right)+\left(1+\epsilon_{h} \epsilon_{\pi}\right)=0,
$$

where

$$
\epsilon=\frac{b \varphi_{2}}{\sqrt{d R_{s}}}, \quad \epsilon_{\pi}=\frac{\pi b}{\sqrt{d R_{s}}}, \quad \epsilon_{h}=\frac{b \varphi_{h}}{\sqrt{d R_{s}}},
$$

with $R_{s}=2 G|M| / c^{2}$. The solutions to the quadratic (3.3) are

$$
\epsilon_{ \pm}=\frac{\epsilon_{h}+\epsilon_{\pi} \pm \sqrt{\left(\epsilon_{h}-\epsilon_{\pi}\right)^{2}-4}}{2} .
$$

It follows that (i) if $\epsilon_{\pi}-\epsilon_{h}>2$ there will be two rays deflected to the observer, (ii) if $\epsilon_{\pi}-\epsilon_{h}<2$ deflection will fully block all rays from reaching the observer, and finally (iii) if $\epsilon_{\pi}-\epsilon_{h}=2$ there will be an umbra region surrounded by a caustic where rays accumulate to produce a brilliant region of highly enhanced intensity [13]. Double imaging in ringholes would tend to occur for generally small mass $|M|$ and mouth separation $d$, and generally large radius of the throat. The effect would also be enhanced when $b$ approaches $a$.

In the above analysis we have assumed that direct rays linking the source to the observer pass on the angular horizon at or near the ringhole's throat. Moreover, it can be shown that if the velocity of the particles coming to the detector is $v$, then the observer will detect a second image of the source with a shifted frequency

$$
\frac{\delta \omega}{\omega}=\frac{\gamma v G|M|}{b\left(\pi-\varphi_{2}\right) c^{2}}
$$

where $\gamma$ is the relativistic factor for velocity $v$, such as it happens in cosmic strings [14].

The lensing and frequency-shifting effects could not occur in wormholes, where one must assume the negative energy to be uniformly or radially distributed in the region surrounding the throat. However, they should happen in ringholes even when the mouths are at rest relative to one another. The latter is the case which is actually assumed in the above calculation and Fig. 1. For an embeddible ringhole, the calculation goes along similar steps, with $\delta=\alpha+\beta$ $(\beta \neq \alpha)$ but, since both $\alpha$ and $\beta$ should be very small, the final result cannot appreciably differ from Eq. (3.4).

\section{B. Discontinuous change of temperature}

If the ringhole is allowed to have its two mouths in relative motion at a speed $V$, setting two objects initially at rest on one side of the throat, but near to it, one on the angular horizon and the other at an angle $2 \pi-\varphi_{h}>\varphi_{2}>\varphi_{h}$, then when the mouth on the other side starts moving, there will appear a transverse velocity component for the object at $2 \pi-\varphi_{h}>\varphi_{2}>\varphi_{h}$ toward the object on the angular horizon due to the diverging effect of negative mass. This velocity component is given by

$$
u=\gamma_{V} V \delta
$$

with $\gamma_{V}$ the relativistic factor for velocity $V$ and $\delta$ as given by Eq. (3.1). Letting the object on $2 \pi-\varphi_{h}>\varphi_{2}>\varphi_{h}$ be a source of radiation and the object on the angular horizon $\varphi_{h}$ an observer, we deduce that the latter must detect a discontinuous change of radiation frequency due to Doppler shift.

In the cosmological context, the ringhole will be backlit by a uniform black body radiation background, so that the Doppler shift would result in a discontinuous change of temperature,

$$
\frac{\delta T}{T}=\frac{\gamma_{V} v G|M|}{b\left(\pi-\varphi_{2}\right) c^{2}},
$$


originated from the motion of one mouth relative to the other that can make the source and observer traverse the throat of the ringhole with moving mouths, in a similar effect to that also induced by cosmic strings [14].

This discontinuous change of temperature would not be expected to happen in wormholes where the throat region is filled with negative energy only and therefore there are no angular horizons.

\section{Line broadening}

On the other hand, assuming the usual Maxwellian velocity distribution, the relative probability for atoms with resonant emission frequency $\omega_{0}[15]$, entering one mouth of a ringhole with its mouths in relative motion at velocity $V$, to have the $Z$ component (we assume $Z$ to be the main axis of the ringhole) of their velocity with values between $v_{Z}$ and $v_{Z}+d v_{Z}$ will be given by

$$
\exp \left[-\frac{m_{A}\left(\pi-\varphi_{2}\right) b c^{3}}{2 k_{B} \gamma_{V} V R_{S} \omega_{0}^{2} T_{M W}}\left(\omega-\omega_{0}\right)^{2}\right] \frac{c d \omega}{\omega_{0}},
$$

where Eq. (2.8) has been used, $m_{A}$ is the mass of the atom, and $T_{\mathrm{MW}}$ is the temperature of the microwave background. So, the lines of the spectrum will have their maximum at $w_{0}$ and a full Doppler-broadened width at half their maximum height

$$
\frac{4 \omega_{0} k_{B} \gamma_{V} V R_{s} T_{\mathrm{MW}} \ln 2}{m_{A} b\left(\pi-\varphi_{2}\right) c^{3}} .
$$

A large broadening would then be expected for spectral lines from atoms emitting through a ringhole embedded in Friedmann space, from values of $\varphi_{2}$ close to $\pi$, but not through any kind of wormholes.

\section{Spectral intensity and luminosity}

Finally, let us consider the effect that spacetime tunnels may have in the spectral intensity of distant objects. If a number $N$ of emitting two-level atoms cross a ringhole, they will pass through some regions with negative energy and some regions with positive energy near the throat. In regions with negative energy chaotically assembled, the temperature will be negative and give rise to population inversion in the energy levels of the atoms, in a direct effect which differs from the pumping by a three-level system that most simply characterizes lasers [15]. Instead, here the population inversion is originated by the fact that in Boltzmann's law

$$
N_{1}=N_{2} \exp \left(\frac{\hbar \omega}{k_{B} T}\right)
$$

(where $N_{1}$ and $N_{2}$ are the population of, respectively, the first and second atomic levels, assumed to be nondegenerate) negative values of $T$ necessarily imply $N_{2}>N_{1}$. In this case, the beam intensity $I$

$$
\frac{\partial I}{\partial Z}=+\left(N_{2}-N_{1}\right) F(\omega)\left(\frac{\hbar \omega B}{c n \Gamma}\right) I
$$

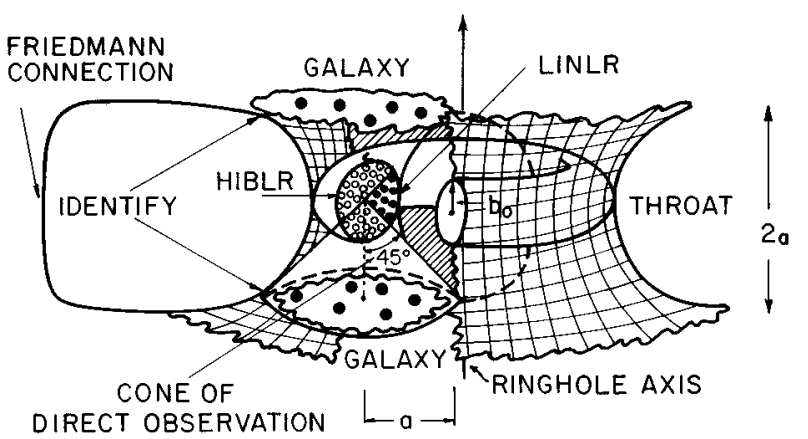

FIG. 2. Schematic picture of a galaxy being observed through a ringhole tunnel with moving mouths. The throat can only be directly accessed if the line of sight lies inside a direct observation cone, $\vartheta<45^{\circ}$. For lines of sight outside that cone, $\vartheta>45^{\circ}$, only the regions placed near the ringhole's mouths and some secondary effects induced on the throat could be observed. On the figure, HIBLR (high-intensity broad-line region) $(\bigcirc)$ and LINLR (lowintensity narrow-line region) $(\mathbf{)})$. The term "Friedmann connection," also on this figure, merely denotes the conventional spacetime that connects the two mouths of the ringhole by going outside the tunnel.

[where [15] $F(\omega) d \omega$ is the fraction of transitions in which the photon frequency lies in a small range $d \omega$ about frequency $\omega, B$ is the Einstein absorption coefficient, $n$ is the refractive index, and $\Gamma$ is the total volume of the considered region] will grow with distance along the ringhole, through the atomic gas. Meanwhile, in the region with positive energy, $N_{1}>N_{2}$, and hence $I$ must decrease with distance along direction $Z$, through the atomic gas. Along a distance $\Delta Z \simeq \sqrt{2} b_{0}$ on the line of sight, one would then expect a large increase of the intensity of the light coming from the compactified regions of galaxies which are confined to a width of the order $b_{0}$, when the given galaxies cross a ringhole's throat.

On the other hand, as far as we are dealing with a ringhole which is embeddible in some region of the universe, the entering of the galaxy in one mouth implies its simultaneous exit at the other mouth [11], so that the overall observable effect will be that of a very brilliant compact region of size $\sim b_{0}$, embedded in the larger galaxy. This effect would be expected for all considered tunnels, though it became most apparent in the case of ringholes because of the existence of angular horizons which make quite neater the separation of the brilliant region from a darker background.

\section{PERSPECTIVE EFFECTS}

All the effects discussed so far refer to the particular case where the observer's line of sight to the object passes through the traversable spacetime tunnels. However, direct access by sight to the region surrounding the ringhole's throat by an observer sustends a given, more or less deformed, cone of direct observation, generated by revolution around an axis parallel to the ringhole axis at the center of a transversal section of the torus, with an angle whose vertex is at the throat. The maximum value of that angle is expected to be (Fig. 2) 


$$
\vartheta \approx \arctan \left(\frac{L_{r}}{2 a}\right)=45^{\circ},
$$

where Eq. (2.9) has been used.

Observers whose line of sight to the throat lies outside that cone will have only a projected evidence of the object's passing through the tunnel throat and, in the case of a static ringhole or in the more realistic case of a ringhole with its mouths set in relative motion with nonzero velocity, they would detect the object reaching the two mouths, as well as some secondary effects induced from the primary effects occurring around the throat (strong broad emission lines, very high luminosities induced by the smallness of the throat, etc.), but not these primary effects themselves. Among the possible secondary effects one may include the existence of regions filled with ionized gas which would be observable whatever angle between the line of sight and the ringhole axis may be formed. These regions should be originated from the high-intensity beam of ionizing photons possessing the whole collection of electronic transition frequencies of the atoms and molecules whose electronic energy levels were inversely populated in the throat region.

It is rather a curious fact that the most remarkable features of the emerging overall picture (see Fig. 2) can, at least qualitatively, be accommodated to the current unified models of galaxies with active nuclei, class 1 and class 2 Seyferts and quasars [16]. These models attribute the differences among these objects to the presence of a dusty torus of dense molecular gas surrounding a black hole. The dusty torus serves as well to give rise to a cone of direct observation with angle of about $45^{\circ}$. Just like for ringholes, all differences can then be attributed to merely being observed by lines of sight which lie inside or outside this cone.

\section{ACKNOWLEDGMENTS}

For useful comments, the author thanks M. Moles of IMAFF. This research was supported by DGICYT under Research Project Nos. PB94-0107 and PB93-0139.
[1] K. Lanczos, Z. Phys. 21, 73 (1924).

[2] W. J. van Stockum, Proc. R. Soc. Edinburgh 57, 135 (1937).

[3] K. Gödel, Rev. Mod. Phys. 21, 447 (1949).

[4] C. W. Misner, in Relativity Theory and Astrophysics I. Relativity and Cosmology, edited by J. Ehlers (American Mathematical Society, Providence, RI, 1967).

[5] M. S. Morris and K. S. Thorne, Am. J. Phys. 56, 395 (1988).

[6] J. Gott, Phys. Rev. Lett. 66, 1126 (1991).

[7] B. Jensen and H. H. Soleng, Phys. Rev. D 45, 3528 (1992).

[8] K. S. Thorne, Black Holes and Time Warps (Norton, New York, 1994).

[9] M. Wisser, Lorentzian Wormholes (AIP, Woodbury, 1996).

[10] M. J. Morris, K. S. Thorne, and U. Yurtsever, Phys. Rev. Lett.
61, 1446 (1988).

[11] P. F. González-Díaz, Phys. Rev. D 54, 6122 (1996).

[12] L. D. Landau and E. M. Lifshitz, The Classical Theory of Fields (Pergamon, Oxford, 1975).

[13] J. G. Cramer, R. L. Forward, M. S. Morris, M. Visser, G. Benford, and G. A. Landis, Phys. Rev. D 51, 3117 (1995).

[14] A. Vilenkin and E. P. S. Shellard, Cosmic Strings and Other Topological Defects (Cambridge University Press, Cambridge, England, 1994).

[15] R. Loudon, The Quantum Theory of Light (Clarendon, Oxford, 1986).

[16] C. M. Urry and P. Padovani, Publ. Astron. Soc. Pac. 107, 803 (1995). 